\title{
Surgical treatment for intrahepatic cholangiocarcinoma
}

\author{
Takahiro Uenishi - Takatsugu Yamamoto • \\ Shigekazu Takemura $\cdot$ Shoji Kubo
}

Received: 23 January 2014/ Accepted: 26 January 2014/Published online: 15 February 2014

(C) Springer Japan 2014

\begin{abstract}
Despite surgical treatment for intrahepatic cholangiocarcinoma (ICC) becoming more widely available, the prognosis after hepatic resection for ICC remains poor. Because ICC is relatively rare, the TNM staging system for ICC was finally established in the 2000s. Resection margin status and lymph node metastases are important prognostic factors after surgery for ICC; however, the true impact of wide resection margins or lymph node dissection on postoperative survival is unclear. Although adjuvant chemotherapy can improve the postoperative prognosis of patients with various types of cancer, no standard regimen has been developed for ICC. Over $50 \%$ of patients suffer postoperative recurrence, even after curative resection, and no effective treatment for recurrent ICC has been established. Therefore, despite advances in imaging studies and hepatobiliary surgery, significant challenges remain in improving the prognosis of patients with ICC.
\end{abstract}

Keywords Intrahepatic cholangiocarcinoma $\cdot$ Surgical treatment · Adjuvant chemotherapy

\section{Introduction}

Although intrahepatic cholangiocarcinoma (ICC) is the second most common primary liver cancer arising from the

T. Uenishi $(\bowtie) \cdot$ T. Yamamoto

Department of Surgery, Ishikiri-Seiki Hospital,

18-28 Yayoi-cho, Higashi-ōsaka, Osaka 579-8026, Japan

e-mail: m6877710@msic.med.osaka-cu.ac.jp

S. Takemura $\cdot$ S. Kubo

Department of Hepato-Biliary-Pancreatic Surgery, Osaka City

University Graduate School of Medicine, Osaka, Japan intrahepatic bile duct epithelium, it only accounts for approximately $4 \%$ of all primary liver cancers in Japan [1]. However, an increase in the incidence and mortality due to ICC over a 30 -year period has been reported in developed countries, including Japan [2-4]. Recent advances in imaging technology and surgical techniques have resulted in hepatic resection for ICC being more frequently performed [5]. Nathan et al. [6] reported a cumulative $34.4 \%$ improvement in the survival of patients who underwent resection for ICC from 1992-2002, but the postoperative outcome of these patients remains unsatisfactory, as the 5-year overall survival rate after potentially curative resection is only 30-40 \% [7-21]. ICC is asymptomatic even at advanced stages, and its associated risk factors in the population are unknown, both of which make its early detection difficult. However, recent epidemiological and pathological studies have suggested that hepatitis $\mathrm{C}$ virus (HCV) infection is closely associated with the development of ICC [22-27]. Kobayashi et al. [28] reported that the cumulative rates of newly diagnosed ICC were $1.6 \%$ at 5 years and $3.5 \%$ at 10 years among 600 patients with HCV-related cirrhosis, which were approximately 1,000 times higher than the rate in the general population of Japan. In fact, Japanese studies have also reported a high prevalence of $\mathrm{HCV}$ infection in patients who underwent resection for ICC, which accounts for $18.8-38.0 \%$ of cases $[1,12,29,30]$. Therefore, surveillance of patients with HCV can increase the likelihood of detecting resectable ICC as well as hepatocellular carcinoma (HCC).

Although surgical resection provides the only chance of long-term survival for patients with ICC, the minimum width of the resection margin required to prevent recurrence is still debated [10,31-34]. ICC often spreads via the lymphatic system and postoperative outcomes for patients 
with lymph node metastases are extremely poor [7-20]; however, to date no consensus among hepatic surgeons has been reached on the indications for surgical treatment of ICC with lymph node metastases and the required extent of lymph node dissection. The extent of both the resection margin and lymph node dissection can be changed by surgeons during hepatic resection for ICC, and it is therefore necessary to clarify their prognostic impact. In a recent meta-analysis of adjuvant therapy for biliary tract cancer, a significant benefit of adjuvant therapy was seen in patients with node-positive or R1 disease [35]. The development of effective adjuvant chemotherapy regimens can help to improve the postoperative outcomes of patients with ICC as many of them have advanced disease. Recently, aggressive treatments, including repeated hepatic resection and radiofrequency ablation (RFA) therapy are increasingly being used to treat recurrent ICC, which may prolong patient survival [21, 36-42]. Despite the many advances in ICC treatment, no standard strategy has been established. This review focuses on the best surgical approach for ICC.

\section{TNM staging systems and prognostic factors}

Despite distinct differences in the biological behavior and postoperative outcomes of HCC and ICC, a distinct TNM staging system for ICC has only recently been established, which is based on data derived from clinical experiences with HCC patients that was used to treat ICC. In 2003, the Liver Cancer Study Group of Japan (LCSGJ) proposed a new staging system for ICC [43] based on data from 245 patients who underwent liver resection for ICC [44]. The LCSGJ staging system was developed only for the massforming (MF) type of ICC and is essentially the same as the HCC staging system, except that serosal invasion includes $\mathrm{T}$ category factors. In 2009, the American Joint Committee on Cancer (AJCC)/International Union against Cancer (UICC) established another staging system for ICC in the seventh edition of their staging manual [45]. This was based on the Surveillance, Epidemiology, and End Results (SEER) database of 598 patients who underwent hepatic resection for ICC [46]. The AJCC/UICC staging system is distinct from that for HCC, as the T category considers the tumor number, vascular invasion, visceral peritoneum perforation or extrahepatic direct invasion, and periductal invasion, but not the tumor size. A number of investigators have analyzed the accuracy of both staging systems in predicting patient survival after hepatic resection for ICC, and proposed some modifications based on their findings [14-16, 47]. Farges et al. [16] reported that the current AJCC/UICC staging system was the only system with good accuracy for predicting disease outcome as the other systems failed to do so, including the system proposed by the
LCSGJ. In contrast, other studies reported that the AJCC/ UICC system could not provide a monotonicity of gradients for the T classification and stage groupings of ICC [14, 47]. Therefore, further studies should be conducted on larger populations to establish an accurate staging system for ICC, since it is essential for the evaluation of treatment and clinical trial outcomes.

Lymph node metastasis is the most important prognostic factor in patients who undergo hepatic resection for ICC [720, 44, 46]. Therefore, in both the AJCC/UICC and LCSGJ staging systems, ICC with nodal metastases is classified as stage IV. Multiple tumors and vascular invasion are T classification components in the AJCC/UICC and LCSGJ systems [43, 45], and the presence of multiple tumors have been reported to strongly influence the survival of patients who underwent hepatic resection for ICC [7-10, 12-14, 19$21,44,46,50]$. In contrast, several studies have also found that aggressive surgical resection for ICC with intrahepatic metastasis confers a survival advantage [17]. Further confirmatory studies are needed to evaluate the effectiveness of hepatic resection for ICC with intrahepatic metastasis. Although vascular invasion was often found to be a prognostic factor according to univariate analysis $[7-13,15,17$, $44,46]$, it was not an independent prognostic predictor in a number of studies [7, 9-11]. Nathan et al. [46] reported that vascular invasion was independently associated with poor postoperative outcomes only in a cohort of patients with NOM0 tumors, as they found that tumor size was not associated with postoperative survival in any analyses that used a cut-off value of 2 or $5 \mathrm{~cm}$; therefore, this parameter was omitted from the AJCC/UICC staging system [45]. Other studies also reported that tumor size was not an independent prognostic factor [7-15, 17-20]. In contrast, the LCSGJ staging system included tumor size, as a Japanese multicenter study showed that a tumor $>2 \mathrm{~cm}$ was an independent prognostic factor for MF-ICC [44]. However, previous studies reported that $\leq 3 \%$ of patients who underwent hepatic resection for MF-ICC had a tumor $\leq 2 \mathrm{~cm}[8,16,46]$, as it is difficult to detect early-stage ICC. The real impact of tumor size on survival is still debated, and it should be evaluated in a much larger population. The LCSGJ proposed that ICC should be subdivided into 3 macroscopic types-the MF type that has a definite mass, the periductal-infiltrating (PI) type that extends along the bile duct, and the intraductal-growth type that is characterized by papillary growth within the ductal lumen and is extremely rare [43]. A number of Ki-ras gene variants have been linked to the PI type rather than the MF type, suggesting that mechanisms of carcinogenesis and clinical characteristics differ between these types [48]. Some investigators reported that postoperative survival of patients with MF and PI type ICC (an MF tumor with periductal invasion) was shorter than that of patients with MF type 
tumors [49-52]. In the AJCC/UICC staging system, ICCs with periductal invasion were classified as T4 tumors [45]. Nevertheless, other studies reported that periductal invasion was not a predictor of poor survival in patients who underwent liver resection for MF-ICC [8, 12-15]. Igami et al. [14] evaluated the validity of the AJCC/UICC staging system for ICC and proposed removing periductal invasion as a $\mathrm{T}$ category determinant. Serosal invasion, together with vascular invasion, is also a $\mathrm{T}$ classification component in the LCSGJ staging system based on an analysis of prognostic factors in 136 patients with MF-ICC [44]. In the AJCC/ UICC staging system, ICCs perforating the visceral peritoneum (serosal invasion) are classified as T3 tumors [45]. However, in previous studies, serosal invasion was not independently associated with postoperative survival [9, 13-15, 50]. Furthermore, our previous study reported that the modified LCSGJ staging system better predicted survival when serosal invasion was removed as a component of $\mathrm{T}$ subgroup determination [15].

Some investigators reported that the serum carbohydrate antigen (CA) 19-9 level was an independent predictor of poor prognosis after hepatic resection for ICC [10, 12, 53]. In our previous study [54], since the serum CYFRA21-1 concentration was related to tumor stage, a high concentration of CYFRA21-1 was an independent prognostic factor associated with tumor recurrence and postoperative death. Adjuvant therapy should be considered for patients with a high serum CA 19-9 or CYFRA21-1 concentration, although new chemotherapeutic regimens with better anticancer activity and a better toxicity profile for patients with ICC still need to be developed.

\section{Lymph node metastasis and dissection}

The prevalence of lymph node metastases detected at surgery for ICC resection has been reported to be 25-50\% [7-10, 12-15, 17-21]. Although lymph node metastasis was found to be an adverse prognostic factor in patients who underwent hepatic resection for ICC [7-15, 17-21], the value of lymph node dissection for ICC is still controversial. Therefore, no standard guidelines for lymph node dissection have been established. Previously, the LCSGJ had proposed that regional lymph nodes should be divided into 3 groups [55] based on several studies of lymphatic spreading routes [56-58]. Group 1 included nodes in the hepatoduodenal ligament, group 2 consisted of nodes along the common hepatic and left gastric artery, around the celiac trunk, or on the posterior surface of the pancreas head, and group 3 included para-aortic nodes. In addition, nodes in the lesser gastric curvature were included in group 1 when the tumor was located in the left hepatic lobe. Therefore, regional lymph node (groups 1 and
2) dissection was commonly performed at hepatobiliary centers in Japan, while lymph node dissection is rarely performed for ICC patients in Western countries. It is important to clarify the benefit of lymph node dissection because surgeons can use it to improve the prognosis of patients with ICC. Some investigators recommended that routine lymph node dissection should be performed because they are frequent metastatic sites [9, 10, 13]. In contrast, Grobmyer et al. [59] suggested that routine lymph node dissection is unnecessary in patients without any clinical or radiological evidence of disease; none of the 38 patients in their study had lymph node metastases when negative imaging studies and negative intraoperative assessments were observed. Choi et al. [9] reported that regional lymph node dissection prolonged the survival of ICC patients with lymph node metastasis, since patients in the regional lymph node dissection group showed similar survival to those in the lymph node sampling group, although there was a significantly higher incidence of lymph node metastases in the former. Conversely, Shimada et al. [60] analyzed the recurrence pattern after resection was performed for ICC, and showed that extended lymph node dissection did not offer any advantage without control of the liver metastases, because most patients who developed recurrence had liver metastases. Inoue et al. [17] suggested that the presence of lymph node metastasis in ICC is a sign of systemic disseminated disease that is incurable by extensive surgery. Some other authors also recommended that patients with nodal metastases should not be considered candidates for extensive surgery, since node dissection does not affect the long-term survival of patients who already have nodal metastases [18, 19]. However, long-term survival after resection for ICC with nodal metastases has been reported, although it is rare [7, 9, 12]. In a recent multicenter analysis [12], only 7 of 139 patients with nodal metastases survived $>4$ years after surgery even if extended dissection beyond the hepatoduodenal ligament had been performed. Therefore, it is important to determine the indications for surgical treatment of ICC with lymph node metastases. Nakagawa et al. [61] suggested that a curative resection with lymphadenectomy improved the survival of patients with a solitary tumor and no more than 2 positive lymph nodes. This was based on their findings that 6 of 23 patients who underwent curative surgery for a solitary tumor with lymph node metastases survived for $>3$ years after surgery, while no patients with both nodal metastases and intrahepatic metastases survived for 3 years. Similarly, another study found that hepatic resection with lymph node dissection may be curative in patients with a single lymph node metastasis and a solitary ICC tumor [62]. In our previous study, we also found that surgery can increase the possibility of long-term survival in patients who have a solitary 
ICC with nodal metastases, as the survival rates for 23 such patients were $35 \%$ at 3 years and $26 \%$ at 5 years, whereas all patients with both lymph node and intrahepatic metastases died within 3 years [7]. Surgery alone cannot prolong survival when both lymph node and intrahepatic metastases are present, and further studies should be conducted to clarify the indication of surgery for ICC with nodal involvement.

\section{Resection margin}

A positive ICC resection margin is closely associated with a poor prognosis [7, 10-13, 15, 17, 19, 20], and resection margin status is a technical concern during ICC surgery. Recent studies have addressed whether the width of the resection margin is related to survival or recurrence after surgery for ICC [10, 31-34]. Ribero et al. [10] reported that the width of a negative margin did not affect postoperative survival because no difference in the survival was noted between patients with a negative margin of $0.5-9 \mathrm{~mm}$ and patients with wider negative margin of $\geq 1 \mathrm{~cm}$. In addition, the margin width was not associated with recurrence rates or the site of recurrence. Tamandl et al. [31] recommended that expected narrow resection margins should not exclude patients from potentially curative surgery, as they found no relationship between resection margin width and recurrence or survival after surgery. In their study, intrahepatic recurrence was observed in $40 \%$ of cases in which a wide margin $(>10 \mathrm{~mm})$ was achieved, $58 \%$ of cases where only a close margin (1-10 mm) was achieved, and in $50 \%$ of cases involving $\mathrm{R} 1$ resection; the incidence of extrahepatic metastasis in each of these scenarios was 27,16 , and $14 \%$, respectively. The median overall survival was 27.2 months in the wide margin group, 29.7 months in the close margin group, and was not reached in the $\mathrm{R} 1$ group. In contrast, Cho et al. [32] reported that every effort should be made to gain a wide resection margin to enhance survival, since they found that a narrow resection margin $(\leq 1 \mathrm{~cm})$ was associated with significantly reduced survival after surgery. We also recommended frozen-section examination of the resection margin to confirm the absence of cancer cells, because tumor cells were frequently found within $5 \mathrm{~mm}$ of the resection margin and their presence was a significant prognostic factor [7]. Recently, Farges et al. [33] showed that in patients who underwent curative resection for ICC without nodal metastases, median survival was closely associated with margin width $(\leq 1 \mathrm{~mm}, 15$ months; 2-4 mm, 36 months; 5-9 mm, 57 months; and $\geq 10 \mathrm{~mm}$, 64 months) and a margin $>5 \mathrm{~mm}$ was an independent predictor of survival. Therefore, they recommended that a margin of at least $5 \mathrm{~mm}$ should be created. Shimada et al. [34] also reported that in patients without nodal involvement, as the margin width increased, there was a linear increase in postoperative survival; a resection margin of $\geq 5 \mathrm{~mm}$ was an independent predictor of survival. In addition, no local recurrences were observed in patients with a wide surgical margin, and intrahepatic recurrence was more frequently observed in patients with a narrow surgical margin. Therefore, they recommended that every effort should be made to achieve a wide surgical margin during hepatic resection for ICC without nodal metastases.

\section{Adjuvant chemotherapy}

Some investigators reported that adjuvant chemotherapy for ICC could prolong postoperative survival [63, 64]. A meta-analysis study also showed a survival benefit of fluorouracil-based adjuvant chemotherapy for cholangiocarcinoma in patients with lymph node-positive disease and $\mathrm{R} 1$ disease [35]. A recent study using a murine model showed that adjuvant gemcitabine chemotherapy after R0resection significantly prolonged the survival of treated animals [65]. However, the benefit of adjuvant chemotherapy on recurrence and survival after resection for ICC is unclear. Although there was no established standard chemotherapy for patients with locally advanced or metastatic biliary tract cancer, the ABC-02 trial [66] showed that cisplatin plus gemcitabine was associated with a significant survival advantage over gemcitabine alone in patients with advanced biliary cancer without any significant increase in toxicity. Wirasorn et al. [67] reported that adjuvant chemotherapy significantly prolonged survival in patients who underwent resection for advanced cholangiocarcinoma, and that patients treated with a combination of gemcitabine and capecitabine had the longest survival time. In Japan, S-1, an oral fluoropyrimidine has been widely used in the treatment of cholangiocarcinoma. Several phase II studies of patients with advanced biliary tract cancer showed that combined gemcitabine and S-1 therapy resulted in a better response rate [68-70]. Murakami et al. [71] suggested that adjuvant gemcitabine and S-1 chemotherapy might improve the outcomes after aggressive surgical resection of advanced biliary carcinoma, as 5-year survival rates of patients who received or did not receive postoperative adjuvant chemotherapy were 57 and $24 \%$, respectively. A prospective randomized trial should be performed to determine the real impact of adjuvant therapy on postoperative outcomes.

\section{Treatments for recurrent ICC}

The postoperative recurrence rate of ICC remains high, and occurs in 50-80\% of cases even after curative 
resection [11, 21, 38-40, 72]. No effective treatments have been established for unresectable or recurrent ICC, and the prognosis of patients with these advanced ICCs is extremely poor. Recently, Ercolani et al. [72] reported that aggressive approaches for recurrent ICC, such as repeated hepatic resection or RFA, could significantly improve prognosis. Although the efficacy of repeated hepatic resection for recurrent $\mathrm{HCC}$ or liver metastases of colon cancer is well documented, there have only been a few reported studies of hepatic resection for recurrent ICC. In a case report by Yamamoto et al. [36], a patient with a solitary ICC recurrence underwent a partial hepatic resection, and no recurrence was observed during a 1-year follow up. Kurosaki et al. [37] reported 2 long-term survivors who underwent repeated hepatic resection for recurrent ICC. Saiura et al. [21] also reported that 3 of 5 patients who underwent hepatic resection for solitary recurrent ICC survived for $>5$ years after initial resection. Ohtsuka et al. [38] suggested that in some cases repeated surgical resection for recurrent ICC can result in longterm survival, as 3 of the 6 patients who underwent complete resection for recurrent ICC were alive and disease-free 32, 39, and 77 months after the second operation. Although RFA is now accepted as an effective and safe treatment for HCC, its potential role in the treatment of ICC is still unclear. In 2002, the first report of a case in which RFA was used to treat cholangiocarcinoma was published [41]. In that report, a patient who received RFA for a single intrahepatic recurrence of cholangiocarcinoma was alive without detectable disease at the 10 months follow-up. Fu et al. [42] reported that RFA provides a treatment option for recurrent ICC in patients who cannot undergo surgery, as the median overall survival was 30 months, and the 1 - and 3 -year survival rates were 87.5 and $37.5 \%$, respectively in 12 patients who underwent RFA for recurrent ICC. Kamphues et al. [39] analyzed the outcome of 13 patients who were treated for recurrent ICC by repeated hepatic resection and/or RFA. In their study, after a median follow-up period of 28 months, 7 were alive with 3 -year survival after primary surgery of $52 \%$. In a recent study of 109 patients treated for recurrent ICC, Zhang et al. [40] compared the outcomes after treatment for recurrent ICC between those treated with repeated resection and those treated with RFA, and found that the median overall survival times were 20.3 and 21.3 months, respectively. There was no significant difference in the overall survival rate, except amongst patients with tumors $>3 \mathrm{~cm}$, who survived longer after repeated resections. Although both repeated hepatic resection and RFA might provide an effective option for recurrent ICC, previous studies had some limitations, such as the lack of a control group or an insufficient number of patients. Therefore, further studies on a larger numbers of patients are needed to confirm the role of both methods in the treatment of recurrent ICC.

\section{Disclosures}

Conflict of Interest: Takahiro Uenishi, Takatsugu Yamamoto, Shigekazu Takemura, and Shoji Kubo declare that they have no conflict of interest.

Human/Animal Rights: All procedures followed were in accordance with the ethical standards of the responsible committee on human experimentation (institutional and national) and with the Helsinki Declaration of 1975, as revised in 2008(5).

Informed Consent: Informed consent was obtained from all patients for being included in the study.

\section{References}

1. Ikai I, Arii S, Okazaki M, Okita K, Omata M, Kojiro M, et al. Report of the 17th Nationwide Follow-up Survey of Primary Liver Cancer in Japan. Hepatol Res. 2007;37:676-91.

2. Khan SA, Thomas HC, Davidson BR, Taylor-Robinson SD. Cholangiocarcinoma. Lancet. 2005;366:1303-14.

3. Okuda K, Nakanuma Y, Miyazaki M. Cholangiocarcinoma: recent progress. Part 1: epidemiology and etiology. J Gastroenterol Hepatol. 2002;17:1049-55.

4. Khan SA, Taylor-Robinson SD, Toledano MB, Beck A, Elliott P, Thomas HC. Changing international trends in mortality rates for liver, biliary and pancreatic tumours. J Hepatol. 2002;37:806-13.

5. Lieser MJ, Barry MK, Rowland C, Ilstrup DM, Nagorney DM. Surgical management of intrahepatic cholangiocarcinoma: a 31-year experience. J Hepatobiliary Pancreat Surg. 1998;5:41-7.

6. Nathan H, Pawlik TM, Wolfgang CL, Choti MA, Cameron JL, Schulick RD. Trends in survival after surgery for cholangiocarcinoma: a 30-year population-based SEER database analysis. J Gastrointest Surg. 2007;11:1488-96.

7. Uenishi T, Kubo S, Yamazaki O, Yamada T, Sasaki Y, Nagano $\mathrm{H}$, et al. Indications for surgical treatment of intrahepatic cholangiocarcinoma with lymph node metastases. J Hepatobiliary Pancreat Surg. 2008;15:417-22.

8. Okabayashi T, Yamamoto J, Kosuge T, Shimada K, Yamasaki S, Takayama $\mathrm{T}$, et al. A new staging system for mass-forming intrahepatic cholangiocarcinoma: analysis of preoperative and postoperative variables. Cancer. 2001;92:2374-83.

9. Choi SB, Kim KS, Choi JY, Park SW, Choi JS, Lee WJ, et al. The prognosis and survival outcome of intrahepatic cholangiocarcinoma following surgical resection: association of lymph node metastasis and lymph node dissection with survival. Ann Surg Oncol. 2009;16:3048-56.

10. Ribero D, Pinna AD, Guglielmi A, Ponti A, Nuzzo G, Giulini SM, et al. Surgical approach for long-term survival of patients with intrahepatic cholangiocarcinoma: a multi-institutional analysis of 434 patients. Arch Surg. 2012;147:1107-13.

11. Yedibela S, Demir R, Zhang W, Meyer T, Hohenberger W, Schönleben F. Surgical treatment of mass-forming intrahepatic cholangiocarcinoma: an 11-year Western single-center experience in 107 patients. Ann Surg Oncol. 2009;16:404-12.

12. Uchiyama K, Yamamoto M, Yamaue H, Ariizumi S, Aoki T, Kokudo N, et al. Impact of nodal involvement on surgical outcomes of intrahepatic cholangiocarcinoma: a multicenter analysis by the Study Group for Hepatic Surgery of the Japanese Society of Hepato-Biliary-Pancreatic Surgery. J Hepatobiliary Pancreat Sci. 2011;18:443-52. 
13. de Jong MC, Nathan H, Sotiropoulos GC, Paul A, Alexandrescu $\mathrm{S}$, Marques $\mathrm{H}$, et al. Intrahepatic cholangiocarcinoma: an international multi-institutional analysis of prognostic factors and lymph node assessment. J Clin Oncol. 2011;29:3140-5.

14. Igami T, Ebata T, Yokoyama Y, Sugawara G, Takahashi Y, et al. Staging of peripheral-type intrahepatic cholangiocarcinoma: appraisal of the new TNM classification and its modifications. World J Surg. 2011;35:2501-9.

15. Uenishi T, Yamazaki O, Yamamoto T, Hirohashi K, Tanaka H, Tanaka S, et al. Serosal invasion in TNM staging of massforming intrahepatic cholangiocarcinoma. J Hepatobiliary Pancreat Surg. 2005;12:479-83.

16. Farges O, Fuks D, Le Treut YP, Azoulay D, Laurent A, Bachellier P, et al. AJCC 7th edition of TNM staging accurately discriminates outcomes of patients with resectable intrahepatic cholangiocarcinoma: by the AFC-IHCC-2009 study group. Cancer. 2011;117:2170-7.

17. Inoue $\mathrm{K}$, Makuuchi M, Takayama T, Torzilli G, Yamamoto J, Shimada $\mathrm{K}$, et al. Long-term survival and prognostic factors in the surgical treatment of mass-forming type cholangiocarcinoma. Surgery. 2000;127:498-505.

18. Nakagohri T, Kinoshita T, Konishi M, Takahashi S, Gotohda N. Surgical outcome and prognostic factors in intrahepatic cholangiocarcinoma. World J Surg. 2008;32(2675-80):19.

19. Isa T, Kusano T, Shimoji H, Takeshima Y, Muto Y, Furukawa M. Predictive factors for long-term survival in patients with intrahepatic cholangiocarcinoma. Am J Surg. 2001;181:507-11.

20. Kawarada Y, Yamagiwa K, Das BC. Analysis of the relationships between clinicopathologic factors and survival time in intrahepatic cholangiocarcinoma. Am J Surg. 2002;183:679-85.

21. Saiura A, Yamamoto J, Kokudo N, Koga R, Seki M, Hiki N, et al. Intrahepatic cholangiocarcinoma: analysis of 44 consecutive resected cases including 5 cases with repeat resections. Am J Surg. 2011;201:203-8.

22. Welzel TM, Graubard BI, El-Serag HB, Shaib YH, Hsing AW, Davila JA, et al. Risk factors for intrahepatic and extrahepatic cholangiocarcinoma in the United States: a population-based case-control study. Clin Gastroenterol Hepatol. 2007;5:1221-8.

23. Yamamoto S, Kubo S, Hai S, Uenishi T, Yamamoto T, Shuto T, et al. Hepatitis $\mathrm{C}$ virus infection as a likely etiology of intrahepatic cholangiocarcinoma. Cancer Sci. 2004;95:592-5.

24. Donato F, Gelatti U, Tagger A, Favret M, Ribero ML, Callea F, et al. Intrahepatic cholangiocarcinoma and hepatitis $\mathrm{C}$ and $\mathrm{B}$ virus infection, alcohol intake, and hepatolithiasis: a case-control study in Italy. Cancer Causes Control. 2001;12:959-64.

25. Shaib YH. Risk factors of intrahepatic cholangiocarcinoma in United States: a case-control study. Gastroenterology. 2005;128: 620-6.

26. Perumal V, Wang J, Thuluvath P, Choti M, Torbenson M. Hepatitis $\mathrm{C}$ and hepatitis $\mathrm{B}$ nucleic acids are present in intrahepatic cholangiocarcinomas from the United States. Hum Pathol. 2006;37:1211-6.

27. Wu TT, Levy M, Correa AM, Rosen CB, Abraham SC. Biliary intraepithelial neoplasia in patients without chronic biliary disease: analysis of liver explants with alcoholic cirrhosis, hepatitis $\mathrm{C}$ infection, and noncirrhotic liver diseases. Cancer. 2009;115:4564-75.

28. Kobayashi M, Ikeda K, Saitoh S, Suzuki F, Tsubota A, Suzuki Y, et al. Incidence of primary cholangiocellular carcinoma of the liver in Japanese patients with hepatitis $\mathrm{C}$ virus-related cirrhosis. Cancer. 2000;88:2471-7.

29. Ariizumi S, Kotera Y, Takahashi Y, Katagiri S, Chen IP, Ota T, et al. Mass-forming intrahepatic cholangiocarcinoma with marked enhancement on arterial-phase computed tomography reflects favorable surgical outcomes. J Surg Oncol. 2011;104:130-9.
30. Hai S, Kubo S, Yamamoto S, Uenishi T, Tanaka H, Shuto T, et al. Clinicopathologic characteristics of hepatitis $\mathrm{C}$ virus-associated intrahepatic cholangiocarcinoma. Dig Surg. 2005;22:432-9.

31. Tamandl D, Herberger B, Gruenberger B, Puhalla H, Klinger M, Gruenberger T. Influence of hepatic resection margin on recurrence and survival in intrahepatic cholangiocarcinoma. Ann Surg Oncol. 2008;15:2787-94.

32. Cho SY, Park SJ, Kim SH, Han SS, Kim YK, Lee KW, et al. Survival analysis of intrahepatic cholangiocarcinoma after resection. Ann Surg Oncol. 2010;17:1823-30.

33. Farges O, Fuks D, Boleslawski E, Le Treut YP, Castaing D, Laurent A, et al. Influence of surgical margins on outcome in patients with intrahepatic cholangiocarcinoma. A multicenter study by the AFC-IHCC-2009 study group. Ann Surg. 2011;254:824-30.

34. Shimada K, Sano T, Sakamoto Y, Esaki M, Kosuge T, Ojima H. Clinical impact of the surgical margin status in hepatectomy for solitary mass-forming type intrahepatic cholangiocarcinoma without lymph node metastases. J Surg Oncol. 2007;96:160-5.

35. Horgan AM, Amir E, Walter T, Knox JJ. Adjuvant therapy in the treatment of biliary tract cancer: a systematic review and metaanalysis. J Clin Oncol. 2012;30:1934-40.

36. Yamamoto M, Takasaki K, Nakano M, Saito A. Hepatic recurrence of cholangiolocellular carcinoma: report of a case. Hepatogastroenterology. 1996;43:1046-50.

37. Kurosaki I, Hatakeyama K. Repeated hepatectomy for recurrent intrahepatic cholangiocarcinoma: report of two cases. Eur J Gastroenterol Hepatol. 2005;17:125-30.

38. Ohtsuka M, Kimura F, Shimizu H, Yoshidome H, Kato A, Yoshitomi H, et al. Significance of repeated resection for recurrent intrahepatic cholangiocarcinoma. Hepatogastroenterology. 2009;56:1-5.

39. Kamphues C, Seehofer D, Eisele RM, Denecke T, Pratschke J, Neumann UP, et al. Recurrent intrahepatic cholangiocarcinoma: single-center experience using repeated hepatectomy and radiofrequency ablation. J Hepatobiliary Pancreat Sci. 2010;17:509-15.

40. Zhang SJ, Hu P, Wang N, Shen Q, Sun AX, Kuang M, et al. Thermal ablation versus repeated hepatic resection for recurrent intrahepatic cholangiocarcinoma. Ann Surg Oncol. 2013;20:3596-602.

41. Slakey DP. Radiofrequency ablation of recurrent cholangiocarcinoma. Am Surg. 2002;68:395-7.

42. Fu Y, Yang W, Wu W, Yan K, Xing BC, Chen MH. Radiofrequency ablation for postoperative recurrences of intrahepatic cholangiocarcinoma. Chin J Cancer Res. 2011;23:295-300.

43. The Liver Cancer Study Group of Japan. General rules for the clinical and pathological study of primary liver cancer. 2nd ed. Tokyo: Kanehara; 2003.

44. Yamasaki S. Intrahepatic cholangiocarcinoma: macroscopic type and stage classification. J Hepatobiliary Pancreat Surg. 2003;10:288-91.

45. Edge SB ed. Intrahepatic bile ducts. AJCC Cancer Staging Manual, 7th ed. New York: Springer; 2009.

46. Nathan H, Aloia TA, Vauthey JN, Abdalla EK, Zhu AX, Schulick $\mathrm{RD}$, et al. A proposed staging system for intrahepatic cholangiocarcinoma. Ann Surg Oncol. 2009;16:14-22.

47. Ribero D, Nuzzo G, Amisano M, Tomatis M, Guglielmi A, Giulini SM, et al. Comparison of the prognostic accuracy of the sixth and seventh editions of the TNM classification for intrahepatic cholangiocarcinoma. HPB (Oxford). 2011;13:198-205.

48. Ohashi K, Nakajima Y, Kanehiro H, Tsutsumi M, Taki J, Aomatsu Y, et al. Ki-ras mutations and p53 protein expressions in intrahepatic cholangiocarcinomas: relation to gross tumor morphology. Gastroenterology. 1995;109:1612-7. 
49. Guglielmi A, Ruzzenente A, Campagnaro T, Pachera S, Valdegamberi A, Nicoli $\mathrm{P}$, et al. Intrahepatic cholangiocarcinoma: prognostic factors after surgical resection. World J Surg. 2009;33:1247-54.

50. Shimada K, Sano T, Sakamoto Y, Esaki M, Kosuge T, Ojima H. Surgical outcomes of the mass-forming plus periductal infiltrating types of intrahepatic cholangiocarcinoma: a comparative study with the typical mass-forming type of intrahepatic cholangiocarcinoma. World J Surg. 2007;31:2016-22.

51. Hirohashi K, Uenishi T, Kubo S, Yamamoto T, Tanaka H, Shuto $\mathrm{T}$, et al. Macroscopic types of intrahepatic cholangiocarcinoma: clinicopathologic features and surgical outcomes. Hepatogastroenterology. 2002;49:326-9.

52. Yamamoto M, Takasaki K, Yoshikawa T, Ueno K, Nakano M. Does gross appearance indicate prognosis in intrahepatic cholangiocarcinoma? J Surg Oncol. 1998;69:162-7.

53. Ohtsuka M, Ito H, Kimura F, Shimizu H, Togawa A, Yoshidome $\mathrm{H}$, et al. Results of surgical treatment for intrahepatic cholangiocarcinoma and clinicopathological factors influencing survival. Br J Surg. 2002;89:1525-31.

54. Uenishi T, Yamazaki O, Tanaka H, Takemura S, Yamamoto T, Tanaka S, et al. Serum cytokeratin 19 fragment (CYFRA21-1) as a prognostic factor in intrahepatic cholangiocarcinoma. Ann Surg Oncol. 2008;15:583-9.

55. Liver Cancer Study Group of Japan. General rules for the clinical and pathological study of primary liver cancer, First English edn. Tokyo: Kanehara; 1997.

56. Tsuji T, Hiraoka T, Kanemitsu K, Takamori H, Tanabe D, Tashiro S. Lymphatic spreading pattern of intrahepatic cholangiocarcinoma. Surgery. 2001;129:401-7.

57. Okami J, Dono K, Sakon M, Tsujie M, Hayashi N, Fujiwara Y, et al. Patterns of regional lymph node involvement in intrahepatic cholangiocarcinoma of the left lobe. J Gastrointest Surg. 2003;7:850-6.

58. Nozaki Y, Yamamoto M, Ikai I, Yamamoto Y, Ozaki N, Fujii H, et al. Reconsideration of the lymph node metastasis pattern $(\mathrm{N}$ factor) from intrahepatic cholangiocarcinoma using the International Union Against Cancer TNM staging system for primary liver carcinoma. Cancer. 1998;83:1923-9.

59. Grobmyer SR, Wang L, Gonen M, Fong Y, Klimstra D, D'Angelica M, et al. Perihepatic lymph node assessment in patients undergoing partial hepatectomy for malignancy. Ann Surg. 2006;244:260-4.

60. Shimada M, Yamashita Y, Aishima S, Shirabe K, Takenaka K, Sugimachi K. Value of lymph node dissection during resection of intrahepatic cholangiocarcinoma. Br J Surg. 2001;88:1463-6.
61. Nakagawa T, Kamiyama T, Kurauchi N, Matsushima M, Nakanishi K, Kamachi H, et al. Number of lymph node metastases is a significant prognostic factor in intrahepatic cholangiocarcinoma. World J Surg. 2005;29:728-33.

62. Suzuki S, Sakaguchi T, Yokoi Y, Okamoto K, Kurachi K, Tsuchiya $\mathrm{Y}$, et al. Clinicopathological prognostic factors and impact of surgical treatment of mass-forming intrahepatic cholangiocarcinoma. World J Surg. 2002;26:687-93.

63. Puhalla H, Schuell B, Pokorny H, Kornek GV, Scheithauer W, Gruenberger T. Treatment and outcome of intrahepatic cholangiocellular carcinoma. Am J Surg. 2005;189:173-7.

64. Liu RQ, Shen SJ, Hu XF, Liu J, Chen LJ, Li XY. Prognosis of the intrahepatic cholangiocarcinoma after resection: hepatitis $B$ virus infection and adjuvant chemotherapy are favorable prognosis factors. Cancer Cell Int. 2013;13:99.

65. Gürlevik E, Fleischmann-Mundt B, Armbrecht N, Longerich T, Woller N, Kloos A, et al. Adjuvant gemcitabine therapy improves survival in a locally induced, R0-resectable model of metastatic intrahepatic cholangiocarcinoma. Hepatology. 2013;58:1034-41.

66. Valle J, Wasan H, Palmer DH, Cunningham D, Anthoney A, Maraveyas A, et al. Cisplatin plus gemcitabine versus gemcitabine for biliary tract cancer. N Engl J Med. 2010;362:1273-81.

67. Wirasorn K, Ngamprasertchai T, Khuntikeo N, Pakkhem A, Ungarereevittaya $\mathrm{P}$, Chindaprasirt $\mathrm{J}$, et al. Adjuvant chemotherapy in resectable cholangiocarcinoma patients. J Gastroenterol Hepatol. 2013;28:1885-91.

68. Sasaki T, Isayama H, Nakai Y, Ito $\mathrm{Y}$, Kogure H, Togawa O, et al. Multicenter, phase II study of gemcitabine and S-1 combination chemotherapy in patients with advanced biliary tract cancer. Cancer Chemother Pharmacol. 2010;65:1101-7.

69. Kanai M, Yoshimura K, Tsumura T, Asada M, Suzuki C, Niimi $\mathrm{M}$, et al. A multi-institution phase II study of gemcitabine/S-1 combination chemotherapy for patients with advanced biliary tract cancer. Cancer Chemother Pharmacol. 2011;67:1429-34.

70. Sasaki T, Isayama H, Nakai Y, Ito Y, Yasuda I, Toda N, et al. A randomized phase II study of gemcitabine and S-1 combination therapy versus gemcitabine monotherapy for advanced biliary tract cancer. Cancer Chemother Pharmacol. 2013;71:973-9.

71. Murakami Y, Uemura K, Sudo T, Hayashidani Y, Hashimoto Y, Nakamura $\mathrm{H}$, et al. Adjuvant gemcitabine plus S-1 chemotherapy improves survival after aggressive surgical resection for advanced biliary carcinoma. Ann Surg. 2009;250:950-6.

72. Ercolani G, Vetrone G, Grazi GL, Aramaki O, Cescon M, Ravaioli M, et al. Intrahepatic cholangiocarcinoma: primary liver resection and aggressive multimodal treatment of recurrence significantly prolong survival. Ann Surg. 2010;252:107-14. 